\title{
Bebidas Mistas de Frutas e Vegetais no Contexto de Alimentação Saudável: uma Revisão de Literatura
}

\section{Mixed Fruit and Vegetable Drinks in the Context of Healthy Eating: a Literature Review}

\author{
Eliane Beatriz Magalhães Silva*a; Jacqueline Aparecida Takahashia; Raquel Linhares Bello de Araújo ${ }^{\mathrm{a}}$ \\ aUniversidade Federal de Minas Gerais, Programa de Pós-Graduação Stricto Sensu em Ciência de Alimentos. MG, Brasil. \\ *E-mail: elianebiamagalhaes@gmail.com
}

\begin{abstract}
Resumo
Este trabalho objetivou realizar uma revisão de literatura sobre bebidas mistas de frutas e vegetais e seus principais componentes no contexto do consumo alimentar. O presente estudo é uma revisão narrativa elaborada a partir da consulta em diferentes bases de dados, como: Web of science e Science Direct. Optou-se pela busca por termos livres. Os termos juice, drinks foram combinados com as palavras fruits "AND" vegetables para localizar estudos sobre os dois temas. Posteriormente, a fim de selecionar estudos com os principais componentes das bebidas mistas, foram utilizados os termos livres "citrus limon", "Malus domestica", "Ananas comosus", "Cucumis savitus", "Brassica oleracea", "Spinacia oleracea", "Zingiber officinale" e "Mentha ssp". O critério de inclusão dos trabalhos foi pautado na seleção de materiais que tivessem por objetivo estudar os efeitos isolados do consumo dos principais ingredientes das bebidas mistas, como também os artigos que utilizaram a bebida mista com alegação "detox" nos estudos. O direcionamento dos artigos selecionados está relacionado com a prospecção da atividade antioxidante e, apesar da composição das bebidas ser diversificada, destacam-se o uso de maçã, de repolho e de pepino como ingredientes das preparações. As bebidas mistas, sucos ou smoothies apresentaram variável composição que determina os atributos sensoriais, composição físico-química, compostos bioativos e atividade antioxidante.
\end{abstract}

Palavras-chave: Suco. Nutrição. Alimentação.

\begin{abstract}
This study aimed to conduct a literature review on mixed fruit and vegetable drinks and their main components in the context of food consumption. The present study is a narrative review elaborated from the consultation in different databases, such as Web of science and Science Direct databases. It was chosen to search free terms. The terms juice, drinks were combined with the words fruits "AND" vegetables to locate studies on both themes. Subsequently, in order to select studies with the main components of mixed drinks, the free terms "citrus limon", "Malus domestica", "Ananas comosus", "Cucumis savitus", "Brassica oleracea”, "Spinacia oleracea", " Zingiber officinale "and "Mentha ssp ". The inclusion criterion of the studies was based on the selection of materials that aimed to study the effects of vegetable consumption alone, which are the main ingredients of mixed drinks and included articles that used the mixed drink or juices with claim "detox" in the studies. The direction of the selected studies is related to the prospecting of antioxidant activity and, although the drinks composition is diverse, the use of apple, cabbage and cucumber as ingredients of the preparations stand out. Plant foods are important sources of nutrients for food. The mixed drinks, juices or smoothies presented variable composition that determines the sensory attributes, physicochemical composition, bioactive compounds and antioxidant activity.
\end{abstract}

Keywords: Juice. Nutrition. Food.

\section{Introdução}

Estabelecer hábitos alimentares saudáveis é um exercício diário pautado em escolhas que garantam o equilíbrio entre ingestão de calorias e, principalmente, a qualidade dos alimentos. Sabe-se que a combinação dos nutrientes é essencial para uma alimentação saudável, na qual os vegetais devem estar presentes (REIS et al., 2017). Isso porque estes apresentam vitaminas, minerais, fibras e compostos bioativos com atividade antioxidante que variam, principalmente, de acordo com os teores de vitamina $\mathrm{C}$, flavonoides e compostos fenólicos (DUZZIONI et al., 2010).

O guia alimentar ressalta a importância de uma alimentação baseada em alimentos frescos, como frutas e legumes, e recomenda diminuir o consumo de alimentos processados e evitar os ultraprocessados. Além disso, a alimentação deve conter quantidades adequadas de nutrientes de acordo com a faixa etária, peso e o nível de atividade física (BRASIL, 2014).

Nesse contexto, a Organização Mundial de Saúde (OMS) lançou a campanha "5 por dia" com recomendações para consumir cinco porções de frutas e vegetais, o que equivale a cerca de $400 \mathrm{~g}$ de frutas e vegetais por dia (ROONEY et al., 2017). Nesse sentido, a inclusão do suco de frutas e vegetais é considerada uma estratégia importante para aumentar o consumo desses alimentos (ZHENG et al., 2017).

$\mathrm{O}$ consumo de bebidas à base de frutas é um hábito comum em vários países e, mesmo que a ingestão seja diferente comparada a uma porção de frutas ou de vegetais, o suco contém os mesmos nutrientes em diferentes proporções (ZHENG et al., 2017). As bebidas preparadas com adição de 
hortaliças consistem em alternativa interessante em relação aos sucos de frutas, por também serem uma fonte dietética de polifenois apresentarem menores teores de açúcares (SPÍNOLA et al., 2017).

Atualmente, o mercado de bebidas à base de frutas e vegetais está em expansão, oferecendo diversos tipos de produtos como sucos e bebidas mistas ou smoothies, com diferentes composições e qualidade (BONG et al., 2017). Há uma tendência no consumo de bebidas gaseificadas doces, que vêm sendo substituídas por bebidas de baixo teor calórico, que ofereçam benefícios nutricionais (BABAJIDE et al., 2013). Outra tendência é a produção de bebidas com processamento mínimo, prensadas a frio e sem aditivos químicos. No entanto, estas possuem o desafio de manter a qualidade microbiológica (SNYDER; WOROBO, 2018).

Um tipo de bebida que se tornou popular nos últimos anos é conhecido como suco verde ou "detox", que são bebidas mistas constituídas por frutas e hortaliças com a suposta função de desintoxicar o organismo e facilitar a perda de peso. Não existe a alegação funcional para o uso dessa denominação "detox" e não está previsto na legislação vigente no Brasil. A utilização do termo em embalagem de produtos pode configurar uma infração sanitária. Dados sobre os efeitos fisiológicos da ingestão deste tipo de bebidas são escassos na literatura, tanto no que se refere a sua composição, extremamente variável no mercado, quanto sobre suas propriedades nutricionais (BONG et al., 2017). Diante do exposto, esta revisão teve como objetivo fazer um levantamento sobre os estudos realizados com bebidas mistas de frutas e vegetais, seus principais componentes no contexto da alimentação saudável e de seus achados.

\section{Desenvolvimento}

\subsection{Metodologia}

O presente estudo é uma revisão narrativa elaborada a partir da consulta em diferentes bases de dados. Foram consultados artigos disponíveis pelo sítio eletrônico de periódicos da CAPES (Coordenação de Aperfeiçoamento de Pessoal de Nível Superior) nas bases de dados Web of Science e Science Direct. Para a realização das buscas, optouse pela consulta por termos livres. Os termos juice, drinks foram combinados com as palavras fruits "AND" vegetables para localizar estudos sobre os dois temas. Posteriormente, a fim de selecionar estudos com os principais componentes das bebidas mistas foram utilizados os termos livres "citrus limon", "Malus domestica", "Ananas comosus", "Cucumis savitus", "Brassica oleracea", "Spinacia oleracea", "Zingiber officinale" e "Mentha ssp".

Como estratégia de busca foram adotados os seguintes critérios de inclusão: artigos publicados entre os anos 2012 e 2018; artigos disponíveis na íntegra; idioma de publicação sendo inglês e português. A seleção dos artigos foi composta por três etapas: inicialmente, pelo título da obra; em seguida, pela análise do resumo e, por último, pela leitura do texto completo. Por fim, realizou-se a análise da metodologia aplicada, resultados e discussão obtidos. Foram excluídos da busca: teses, dissertação e artigos de revisão.

$\mathrm{Na}$ busca foram encontrados 1849 artigos, dos quais 10 foram selecionados e tinham como tema estudos de análise das bebidas mistas (06 artigos) e bebidas e saúde em estudo in vivo ou in vitro (04 artigos). Sete artigos sobre os componentes das bebidas foram selecionados. Os artigos foram separados e seus dados organizados de acordo com as temáticas, totalizando 17 artigos selecionados.

Com os dados coletados, foi realizado um breve histórico das bebidas mistas no conceito da alimentação saudável e, posteriormente, foram apresentados os principais componentes destas bebidas. Os resultados foram apresentados de forma qualitativa.

\subsection{Discussão}

\subsubsection{Bebidas mistas de frutas e vegetais no contexto da alimentação}

A dieta de desintoxicação ou detox é uma dieta líquida baseada em sopas, sucos mistos de frutas e vegetais e chás, vendida em forma de kits para programas de dietas. Estes são utilizados como intervenções de curto prazo, que geralmente variam entre 48 horas até 21 dias e têm como objetivo promover eliminação de toxinas e perda de peso (KLEIN; KIAT, 2015). Contudo, estudos científicos que sustentem essas alegações são escassos (OBERT et al., 2017). Tais estratégias populares se baseiam na justificativa de que o organismo oxidado precisaria eliminar toxinas acumuladas por meio de dietas hipocalóricas. Com a popularidade das dietas de desintoxicação, sugere-se que deveria ser implementada legislação para informar os consumidores e profissionais da saúde sobre informações não comprovadas (MARTÍN et al., 2017), pois a falta de regulamentação da rotulagem e alegações dos produtos de desintoxicação é uma grande preocupação (KLEIN; KIAT, 2015).

Esse tipo de bebida não tem uma receita padrão, mas em geral, é preparado com uma mistura de frutas, usualmente maçã, limão e vegetais, como gengibre, pepino, couve, brócolis e espinafre. A combinação de componentes como esses em bebidas mistas se baseia no fato de serem ricos em diferentes ingredientes com ação benéfica para a saúde, mas não necessariamente detoxificantes ou emagrecedores. $\mathrm{O}$ conhecimento das características nutricionais dos componentes das bebidas detox é importante para esclarecer aos consumidores suas características nutricionais (OLIVEIRA et al., 2013). Dessa forma, foram pesquisados dados nutricionais e propriedades biológicas de alguns dos principais componentes (limão, maçã, pepino e couve) desse tipo de bebida mista. 


\subsubsection{Principais ingredientes das bebidas mistas de frutas e vegetais}

\subsubsection{Limão (Citrus limon)}

Citrus é o gênero mais importante de árvores frutíferas e o limão é destaque, pois tem um forte valor comercial para o mercado de produtos frescos e para a indústria alimentícia (GONZÁLEZ-MOLINA et al., 2010). O limão e seus derivados têm ampla aceitação no mercado sendo usado em alimentos, fármacos, chás e refrigerantes (JAYAPRAKASHA et al., 2013).

Os efeitos e propriedades que promovem a saúde foram associados aos seus conteúdos, principalmente a vitamina C, que tem papel importante na prevenção de doenças. Além deste micronutriente, relata-se a presença de flavonoides, limonoides, cumarinas e carotenoides (DAHMOUNE et al., 2013), que possuem ação anti-inflamatória em função de sua composição fitoquímica (SRIDHARAN et al., 2016). Também possui propriedade antibacteriana, antiviral, antioxidante, antifúngica, analgésica (JAISWAL et al., 2015). Esses componentes atuam de forma sinérgica em relação as suas atividades biológicas (GONZÁLEZ-MOLINA et al., 2010). Campêlo et al. (2011) relatam potente efeito protetor usando o óleo essencial de $C$. limon como antioxidante em ratos com distúrbios cerebrais, diminuindo a peroxidação lipídica. Um efeito hepatoprotetor foi observado por Jaiswal et al. (2015) em estudo com ratos expostos ao pesticida carbofurano, submetidos a pré-tratamento com extrato de limão.

Os efeitos comportamentais em ratos foram avaliados por Khan e Riaz (2015). Neste estudo, os ratos foram suplementados com Citrus limon em três doses diferentes: $0,2,0,4$ e $0,6 \mathrm{~mL} / \mathrm{kg}$, classificadas como baixas, moderadas e altas doses. As atividades ansiolíticas e antidepressivas foram especificamente avaliadas duas vezes durante 15 dias, utilizando teste de campo aberto, labirinto em cruz elevado e teste de natação forçada. Os resultados sugeriram que $C$. limon na dose moderada tenha efeito ansiolítico.

\subsubsection{Maçã (Malus domestica)}

A maçã é fonte de monossacarídeos, minerais, fibras e ácidos orgânicos, como o ácido málico. É rica em compostos fenólicos e vitamina $\mathrm{C}$ concentrados, que estão presentes inclusive na casca e no bagaço (FERRENTINO et al., 2018; WRUSS et al., 2015). O consumo da maçã está associado à prevenção de doenças como diabetes tipo II e doenças cardiovasculares (KALINOWSKA et al., 2014).

A composição química de maçãs depende do tipo da maturação das frutas, estando associada à sua qualidade nutricional e sensorial, tais como: o sabor e a cor (ERCOLI et al., 2017). O processamento de maçãs durante a produção de suco reduz significativamente seu conteúdo fenólico e a atividade antioxidante, que também podem mudar durante o armazenamento como resultado da degradação oxidativa e não oxidante. Estes fatores podem alterar a quantidade final de nutrientes da fruta ou suco que será absorvida na alimentação (CANDRAWINATA et al., 2013).

Sampath et al. (2017) realizaram a suplementação de floretina ou 6-gingerol, compostos fenólicos encontrados na maçã e no gengibre, em duas doses diferentes para camundongos C57BL em seis grupos diferentes com 11 animais cada, alimentados com dieta rica em gordura ou dieta padrão por um período de 17 semanas. Os camundongos dos grupos da suplementação apresentaram redução significativa da glicose plasmática, alanina aminotransferase, aspartato aminotransferase, sugerindo que a maçã e o gengibre são potenciais compostos dietéticos, que podem aliviar as complicações induzidas pelo diabetes.

\subsubsection{Abacaxi (Ananas comosus)}

O abacaxi é uma fruta tropical que possui várias propriedades medicinais e se destaca pelos compostos bioativos, entre esses, ananasato, $\beta$-sitosterol e bromelina (KARGUTKAR; BRIJESH, 2018) vitamina C, $\beta$-caroteno, compostos fenólicos e flavonoides (HOSSAIN; RAHMAN, 2011). É popularmente consumido como fruta fresca, ou em sucos e geleias, por apresentar aroma e sabor agradáveis (DIFONZO et al., 2019).

O abacaxi é utilizado de forma integral na tecnologia de alimentos. Baseado nessa informação, Hajar et al. (2012) estudaram as propriedades físico-químicas do extrato de casca de abacaxi em três diferentes estágios de maturação e verificaram aumento de 5\% no teor de polpa com o aumento do nível dos estádios de maturação, o que pode contribuir para maior desenvolvimento no setor agrícola. Para avaliar o efeito do consumo da fruta, Cervo et al. (2014) estudaram o consumo de abacaxi em conserva em 89 crianças em idade escolar e observaram uma diminuição na incidência de infecções virais e bacterianas em dois grupos (normal e baixo peso).

\subsubsection{Pepino (Cucumis savitus)}

O pepino é composto por $95 \%$ de água, sendo relativamente rico em fibras, característica adequada para o bom funcionamento do sistema digestivo. O pepino é pouco calórico e contém pequenas quantidades de vitamina $\mathrm{C}$, folato, potássio e vitamina $\mathrm{A}$, contida na casca, que geralmente não é aproveitada (CARVALHO et al., 2013). O pepino é consumido em saladas ou fermentado (picles) (MUKHERJEE et al., 2013).

Possui compostos fenólicos, que têm um papel na prevenção de doenças cardiovasculares e atividade anticancerígena (ZHAO et al., 2014). Além de ser utilizado na alimentação, é matéria-prima para fabricação de cosméticos (MUKHERJEE et al., 2013).

Nash et al. (2018) estudaram o efeito de um extrato aquoso do pepino em um ensaio clínico randomizado, duplocego, de grupos paralelos, com 122 pacientes (56 homens e 66 mulheres) com diagnóstico de osteoartrite moderada do joelho. 
No grupo em que usaram o pepino, o escore da doença foi reduzido em $22 \%$ e $70 \%$ nos dias 30 e 180 , respectivamente, em comparação com uma diminuição de $14 \%$ e $32 \%$ no grupo com medicamento controle.

\subsubsection{Couve (Brassica oleracea)}

A couve é um vegetal verde escuro, sendo fonte de minerais, principalmente $\mathrm{K}, \mathrm{Ca}, \mathrm{Mg}$ e fibras e compostos bioativos como polifenois e glucosinatos que protegem o corpo humano contra danos causados por espécies reativas do oxigênio (ERO) (JEON et al., 2018; THAVARAJAH et al., 2016).

Pode-se destacar entre os nutrientes, os altos níveis de ômega 3 e pequenas quantidades de fitoesteroides. Esses componentes são benéficos na redução das lipoproteínas de baixa densidade plasmática e colesterol, que são fatores de risco para o desenvolvimento de doenças cardiovasculares (VIDAL et al., 2018).

O consumo da couve também está associado à redução de N-nitrosodimetilamina emergente, um composto carcinogênico derivado de alimentos ricos em nitratos e compostos de amina (BIEGAŃSKA-MARECIK et al., 2017). Thavarajah et al. (2016) determinaram a composição nutricional da couve e mostram que cinco folhas de couve fresca podem fornecer porcentagem significativa da ingestão diária recomendada de micronutrientes minerais $(188-873 \mathrm{mg}$ $\mathrm{K}$; 35-300 mg Ca; 20-100 mg).

Em função da importância dos compostos bioativos presentes nas hortaliças, Lin e Harnly (2009) realizaram um estudo abrangente utilizando cromatrografia líquida e espectrometria de massas para identificar componentes fenólicos de couve e brócolis chinês, vegetais de folhas verdes do gênero Brassica, que levou à identificação de 45 flavonoides e 13 derivados do ácido hidroxicinâmico. A identificação foi baseada na comparação de compostos previamente relatados na literatura para esses tipos de vegetais.

Os vegetais de folhas verdes, incluindo a couve, têm efeitos hipocolesterolêmicos, redução da absorção do colesterol, tem um efeito inibitório do crescimento celular anormal e inibição da atividade da 3-hidroxi-3-metilglutaril-coenzima A (HMGCoA) redutase (JEON et al., 2018).

Kushimoto et al. (2018) investigaram a capacidade da suplementação dietética com suco de couve para retardar o declínio cognitivo no camundongo com propensão ao envelhecimento acelerado pela senescência. Os camundongos foram alimentados com uma dieta contendo $0,8 \%(\mathrm{p} / \mathrm{p})$ de suco de couve ou uma dieta de controle durante 16 semanas, e o desempenho cognitivo foi examinado. Observaram que a suplementação de couve dietética pode suprimir o declínio cognitivo e o dano oxidativo relacionado à idade por meio da ativação de aumento significativo na transcrição gênica e na expressão proteica da proteína de choque térmico (HSP70) em camundongos.

\subsubsection{Espinafre (Spinacia oleracea)}

Espinafre é vegetal folhoso verde escuro, membro da família da Amaranthaceae, rico em $\beta$-caroteno, luteína, flavonoides, glicolipídeos, derivados de ácido cumárico e $\alpha$-tocoferol, sendo associado a efeitos promotores de saúde (SYAMILA et al., 2019). Esses fitoquímicos bioativos são capazes de atuar contra espécies reativas de oxigênio e de modular a expressão de gênica de proteínas que desempenham ação de modulação do metabolismo dos seres humanos (FIORITO et al., 2019). Além de ser consumido em forma de saladas ou cozido após tratamento térmico, está se tornando cada vez mais popular a ingestão de espinafre em formas liquefeitas não aquecidas, como suco e smoothie.

Em estudo, Chung et al. (2019) compararam os efeitos dos métodos de cocção mais comuns, fervura e vapor, cru e na forma de suco batido, sob condições realistas na liberação de luteína de folhas de espinafre, concluindo que o maior rendimento de luteína liberada foi obtido a partir de espinafre liquefeito.

\subsubsection{Gengibre (Zingiber officinale)}

O gengibre é uma especiaria cujo rizoma é amplamente comercializado em função de seu emprego alimentar e industrial, especialmente como matéria-prima para fabricação de bebidas, perfumes e produtos de confeitaria como pães, bolos, biscoitos e geleias (LIMA et al., 2014).

O gingerol é o componente bioativo principal, sendo este constituinte responsável pelo sabor picante do gengibre (REIS et al., 2017). Estudo realizado por Lima et al. (2014) corroboram que os compostos bioativos presentes no gengibre possuem efeitos positivos nos indivíduos com diabetes tipo II, diminuindo o estresse oxidativo e o quadro de hiperglicemia, os quais podem estar associados aos compostos fenólicos, que atuam degradando o excesso de radicais livres produzidos no paciente diabético.

Ebrahimzadeh et al. (2016) realizaram um estudo com 80 mulheres obesas (de 18 a 45 anos) com o índice de massa corporal (IMC) de $\left(30-40 \mathrm{~kg} / \mathrm{m}^{2}\right)$ que foram distribuídas aleatoriamente em grupos de gengibre ou placebo (recebendo $2 \mathrm{~g} /$ dia de pó de gengibre ou amido de milho como dois comprimidos de $1 \mathrm{~g}$ por 12 semanas). O IMC e a composição corporal foram avaliados a cada quatro semanas, e os níveis séricos de leptina, adiponectina, resistina, insulina e glicose foram determinados antes e após a intervenção. Os resultados revelaram que o consumo de gengibre resultou em uma diminuição ligeira, mas estatisticamente significativa no IMC, sendo valor médio de $34,34 \mathrm{~kg} / \mathrm{m}^{2}$ para o grupo que ingeriu o gengibre e $35,46 \mathrm{~kg} / \mathrm{m}^{2}$ para o grupo placebo.

\subsubsection{Hortelã (Mentha ssp.)}

O gênero Mentha compreende cerca de 30 espécies que se desenvolvem em diversas regiões da Europa, Ásia, Austrália e América do Sul e, entre essas espécies se destaca a Mentha 
arvensis. Na hortelã, o composto majoritário é o mentol, um monoterpeno que é usado para conferir sabor e aroma de menta a remédios, balas, creme dental e outros produtos da indústria de alimentos, cosmética e farmacêutica (REIS et al., 2017). A hortelã é usada em diferentes tratamentos medicinais tradicionais como remédios de ervas e na indústria de alimentos como aditivos alimentares e potenciadores de sabor por causa de suas propriedades olfativas (BENABDALLAH et al., 2016). Possui componentes bioativos como quercetina, vitamina $\mathrm{K}$, eugenol e timol. Contém monoterpenos, ácidos fenólicos, taninos e minerais (NRIPENDRA et al., 2013).

\subsubsection{Estudos clínicos com componentes de bebidas mistas}

Nesta etapa, foram selecionados os estudos que foram realizados com as frutas e vegetais que são utilizados como componentes das bebidas mistas, conforme apresentado no Quadro 1.

Quadro 1 - Estudos clínicos com os componentes das bebidas mistas

\begin{tabular}{|c|c|c|c|c|}
\hline Ingredientes & Design do estudo & Objetivos & Resultados & $\begin{array}{c}\text { Autores } \\
\text { (ano) }\end{array}$ \\
\hline Limão & $\begin{array}{c}\text { Ensaio clínico randomizado } \\
\text { controlado de delineamento } \\
\text { paralelo, } \\
112 \text { pacientes hiperlipidêmicos } \\
\text { de } 30 \text { a } 60 \text { anos. }\end{array}$ & $\begin{array}{l}\text { Avaliar o efeito do limão no } \\
\text { perfil bioquímico de pacientes } \\
\text { hiperlipidêmicos. }\end{array}$ & $\begin{array}{l}\text { Ocorreu uma diminuição } \\
\text { significativa no colesterol total, } \\
\text { lipoproteína de baixa densidade- } \\
\text { colesterol e fibrinogênio. }\end{array}$ & $\begin{array}{l}\text { Aslani et } \\
\text { al. (2016) }\end{array}$ \\
\hline $\begin{array}{l}\text { Maça e } \\
\text { espinafre }\end{array}$ & $\begin{array}{l}\text { Estudo randomizado, } \\
\text { controlado, cruzado } \\
\text { comhomense mulheres } \\
\text { saudáveis }(n=30) \text {. }\end{array}$ & $\begin{array}{c}\text { Investigar os efeitos } \\
\text { independentes e aditivos de } \\
\text { maçãs ricas em flavonóidese } \\
\text { espinafre rico em nitrato no } \\
\text { estado do óxidonítrico, função } \\
\text { endotelial e pressão arterial. }\end{array}$ & $\begin{array}{l}\text { Houve maior dilatação mediada } \\
\text { pelo fluxo }(P<0,05) \text { e menor } \\
\text { pressão de pulso }(P<0,05) \text {, e maçã } \\
\text { e espinafre resultaram em menor } \\
\text { pressão arterial sistólica }(P<0,05) \text {. }\end{array}$ & $\begin{array}{l}\text { Bondonno } \\
\text { et al. } \\
\text { (2012) }\end{array}$ \\
\hline Gengibre & $\begin{array}{c}\text { Estudo randomizado, } \\
\text { experimental e controlado. } \\
30 \text { mulheres saudáveis com } \\
\text { sobrepeso e idade entre } 20 \text { e } 30 \\
\text { anos. }\end{array}$ & $\begin{array}{l}\text { Avaliar o suplemento de gengibre } \\
\text { e o treinamento intervalado de } \\
\text { alta intensidade (HIIT) robre } \\
\text { os índices inflamatórios que } \\
\text { contribuem para a ateroscleros } \\
\text { em mulheres rom } \\
\text { excesso de peso. }\end{array}$ & $\begin{array}{l}\text { Ocorreuumadiminuiçãosignificativa } \\
\text { na densidade de proteína quimiótica } \\
\text { tática de monócitos tipo1 (MCP-1) } \\
\text { nos grupos HIIT+ gengibre e HIIT + } \\
\text { placebo. A análise de variância não } \\
\text { mostrou diferenças significativas. }\end{array}$ & $\begin{array}{l}\text { Nayebifar } \\
\text { et al. } \\
(2016)\end{array}$ \\
\hline Couve & $\begin{array}{l}\text { Estudo com } 84 \text { pacientes } \\
\text { hipertensos subclínicos } \\
\text { receberam } 300 \mathrm{~mL} / \text { dia de suco } \\
\text { de couve por } 6 \text { semanas. }\end{array}$ & $\begin{array}{l}\text { Examinar se a suplementação } \\
\text { diária de suco de couvepode } \\
\text { modular a pressão arterial (PA), } \\
\text { os níveis de perfil lipídico e a } \\
\text { glicemia, e se essa modulação } \\
\text { pode ser afetada por um grupo } \\
\text { de enzimas com polimorfismo } \\
\text { genético relacionadas ao } \\
\text { extresse oxidativo. }\end{array}$ & $\begin{array}{l}\text { Ocorreu melhoria no perfil lipídico } \\
\text { e glicemia em pacientes hipertensos } \\
\text { subclínicas dependendo do tipo } \\
\text { de enzimas com polimorfismos } \\
\text { genéticos. }\end{array}$ & $\begin{array}{c}\text { Han et al. } \\
\quad(2015)\end{array}$ \\
\hline Pepino & $\begin{array}{l}\text { Ensaio clínico randomizado, } \\
\text { duplo- cego, de grupos } \\
\text { paralelos, } 122 \text { pacientes ( } 56 \\
\text { homens e } 66 \text { mulheres) - idade } \\
\text { entre } 40 \text { e } 75 \text { anos e } \\
\text { diagnóstico de } \\
\text { Osteoartrite OA moderada do } \\
\text { joelho. }\end{array}$ & $\begin{array}{l}\text { Avaliar a eficácia do Q-Actin } \\
\text { TM, um extrato aquoso de } \\
\text { Cucumis sativus (pepino; } \\
\text { CSE) contra glucosamina- } \\
\text { condroitina (GC) no manejo da } \\
\text { OA moderada do joelho. } \\
\text { A intervenção de } 180 \text { dias. }\end{array}$ & $\begin{array}{l}\text { No grupo que usou o pepino, o } \\
\text { escore da doença foi reduzido } \\
\text { em } 22 \% \text { e } 70 \% \text { nos dias } 30 \text { e } 180 \text {, } \\
\text { respectivamente, em comparação } \\
\text { com uma diminuição de } 14 \% \text { e } \\
32 \% \text { no grupo com medicamento. } \\
\text { Tendên- cias semelhantes foram } \\
\text { observadas para todos os outros } \\
\text { escores de dor. }\end{array}$ & $\begin{array}{l}\text { Nash et } \\
\text { al. } \\
(2018)\end{array}$ \\
\hline Menta & $\begin{array}{c}\text { Ensaio clínico randomizado, } \\
\text { duplo-cego, controlado com } \\
\text { placebo, } 50 \text { pacientes com } \\
\text { dispepsia funcional foram } \\
\text { comparados com aqueles de um } \\
\text { grupo placebo }(\mathrm{n}=50) . \\
\end{array}$ & $\begin{array}{c}\text { Estudar a eficácia e segurança } \\
\text { do } M \text {. Pulegium no tratamento } \\
\text { de pacientes com dispepsia } \\
\text { funcional. }\end{array}$ & $\begin{array}{l}\text { O extrato reduziu significativamente } \\
\text { infecção por } H \text {. pylori determinada } \\
\text { pelo teste da urease. }\end{array}$ & $\begin{array}{l}\text { Khonche } \\
\text { et al. } \\
\text { (2017) }\end{array}$ \\
\hline Abacaxi & $\begin{array}{c}\text { Estudo randomizado e } \\
\text { controlado com noventa e oito } \\
\text { (98) escolares com idade média } \\
\text { de } 8,44 \text {. }\end{array}$ & $\begin{array}{c}\text { Examinar os } \\
\text { Efeitos do consumo de } \\
\text { abacaxi em conserva na } \\
\text { imunomodulação, no estado } \\
\text { nutricional e na saúde física de } \\
\text { crianças em idade escolar. }\end{array}$ & $\begin{array}{l}\text { Houve diminuição na incidência de } \\
\text { infecções virais e bacterianas para } \\
\text { o grupo (normal e baixo peso) após } \\
\text { o consumo de abacaxi em conserva. } \\
\text { A produção de granulócitos } \\
\text { aumentou para indivíduos com peso } \\
\text { normal e para baixo peso. }\end{array}$ & $\begin{array}{l}\text { Cervo et } \\
\text { al. } \\
(2014)\end{array}$ \\
\hline
\end{tabular}




\subsubsection{Estudos da composição fisico-química e potencial} antioxidante das bebidas mistas

No Quadro 2 estão descritas informações nutricionais das bebidas mistas dos estudos selecionados. Observou-se um direcionamento dos estudos para análise da atividade antioxidante.

Quadro 2 - Tipos de bebidas, análises e objetivos dos estudos

\begin{tabular}{|c|c|c|c|}
\hline Tipo de Bebida & Tipo de Avaliação & $\begin{array}{l}\text { Objetivos } \\
\end{array}$ & Autores (ano) \\
\hline Sucos em pó & $\begin{array}{l}\text { Atividade antioxidante } \\
\text { e análise sensorial. }\end{array}$ & $\begin{array}{l}\text { Otimizar a proporção de pós de brócolis, repolho e cenoura para } \\
\text { o desenvolvimento de pós de suco com grandes quantidades de } \\
\text { compostos fenólicos. }\end{array}$ & $\begin{array}{l}\text { Kim et al. } \\
\text { (2016) }\end{array}$ \\
\hline $\begin{array}{l}\text { Suco líquido } \\
\text { pasteurizado }\end{array}$ & $\begin{array}{l}\text { Caracterização físico- } \\
\text { química e atividade } \\
\text { antioxidante }\end{array}$ & $\begin{array}{l}\text { Desenvolver uma nova bebida de frutas condimentada a partir da } \\
\text { mistura de abacaxi, pepino, cravo e gengibre. }\end{array}$ & $\begin{array}{l}\text { Babajide et al. } \\
\quad \text { (2013) }\end{array}$ \\
\hline $\begin{array}{l}\text { Suco líquido } \\
\text { pasteurizado }\end{array}$ & Atividade antioxidante & $\begin{array}{l}\text { Determinar os compostos ativos (vitamina C, polifenois e } \\
\text { glicosilados) e uma bebida à base de suco de maçã com adição de } \\
\text { folhas de couve congelada e liofilizada, fresca e pós tratamento } \\
\text { térmico. }\end{array}$ & $\begin{array}{l}\text { Bieganska- } \\
\text { Marecik } \\
\text { et al. }(2017)\end{array}$ \\
\hline Suco líquido coado & Atividade antioxidante & $\begin{array}{l}\text { Determinar o efeito antioxidante do suco verde em comparação } \\
\text { com o suco de laranja. }\end{array}$ & $\begin{array}{l}\text { Oliveira et al. } \\
\quad(2013)\end{array}$ \\
\hline $\begin{array}{l}\text { Suco batido no } \\
\text { liquidificador } \\
\text { ou processado } \\
\text { descartando o } \\
\text { resíduo da polpa }\end{array}$ & $\begin{array}{l}\text { Caracterização físico- } \\
\text { química }\end{array}$ & $\begin{array}{l}\text { Investigar a composição do oxalato suco verde preparado em } \\
\text { diferentes processos. }\end{array}$ & $\begin{array}{l}\text { Vanhanen e } \\
\text { Savage }(2015)\end{array}$ \\
\hline Smoothies de frutas & $\begin{array}{l}\text { Características físico- } \\
\text { química }\end{array}$ & $\begin{array}{l}\text { Caracterizar as propriedades físico- químicas, termo físicas e } \\
\text { reológicas de amostras de smoothies de frutas }\end{array}$ & $\begin{array}{l}\text { Moura et al. } \\
\quad(2017)\end{array}$ \\
\hline
\end{tabular}

Fonte: Dados da pesquisa

As formulações das bebidas se mostraram variáveis. Biegańska-Marecik et al. (2017) usaram couve congelada (13\%) e couve liofilizada (3\%) em suco de maçã. Vanhanen e Savage (2015) usaram $300 \mathrm{~g}$ e $600 \mathrm{~g}$ de espinafre em suco centrifugado. Babajide et al. (2013) desenvolveram uma bebida com $50 \%$ de abacaxi e $50 \%$ de pepino e diferentes concentrações de especiarias. Kim et al. (2016) estudaram treze sucos em diferentes proporções de frutas e vegetais visando melhorar a aceitação e atividade antioxidante dos mesmos. Pode-se destacar, entre os dez estudos, a maçã como a fruta mais utilizada.

Entre os aspectos analisados pelos autores que estudaram suco verdes/detox, um ponto importante discutido foi o modo de conservação, já que este influencia sua composição, podendo ocorrer redução da qualidade quando armazenado em temperatura ambiente. Esse fato deve ser considerado pelas indústrias ao desenvolverem esse tipo de suco, já que pode haver perda das principais características nutricionais e sensoriais.

Moura et al. (2017) analisaram pH, atividade de água, atividade antoxidante (DPPH), densidade, sólidos solúveis, cor, comportamento reológico, propriedades térmicas e sensoriais de smoothies verdes armazenados sob refrigeração e à temperatura ambiente. Observaram redução, a partir de 49 dias de armazenamento, nos teores de compostos bioativos, alteração de cor, sabor e odor daqueles acondicionados à temperatura ambiente. Em estudo sobre compostos bioativos do limão, González-Molina et al. (2010) destacaram que a presença de oxigênio e luz, a temperatura de armazenamento e o tempo de armazenagem afetam a integridade do ácido ascórbico nos sucos de cítricos.

Hornedo-Ortega et al. (2017) avaliando uma bebida fermentada de morango, relataram que as antocianinas e a atividade antioxidante foram reduzidas com o tempo e de acordo com a temperatura de armazenamento dos produtos, sendo que, em temperaturas de $4^{\circ} \mathrm{C}$, conservaram-se por maior tempo do que à temperatura ambiente $\left(20^{\circ} \mathrm{C}\right)$. Dionisio et al. (2016), ao estudarem a estabilidade de uma bebida funcional pasteurizada à base de yacon e frutas tropicais (camu-camu, acerola, caju, cajá, açaí e abacaxi), observaram a estabilidade sensorial e microbiológica da bebida refrigerada a $5{ }^{\circ} \mathrm{C}$ até 90 dias de armazenamento e redução de aproximadamente $20 \%$ da atividade antioxidante total após 225 dias de armazenamento, indicando perdas importantes para esta propriedade na bebida.

As bebidas mistas podem ser enriquecidas com fibra insolúvel principalmente pela adição de vegetais folhosos, o que pode ser interessante para a melhora da função intestinal. Porém, estas são ricas em oxalato, que é um anti-nutriente potencialmente tóxico à saúde humana, encontrado em altos níveis no espinafre e em níveis moderados em outros vegetais folhosos. Este importante aspecto foi abordado por Vanhanen e Savage (2015) para verificar a relação entre o tipo de processamento do suco em relação às fibras e a quantidade de oxalato em suco verde com a análise cromatográfica por (HPLC) mostraram que a remoção do resíduo das folhas que contém a maior parte das fibras, não diminuiu os níveis de oxalato solúvel na bebida. Portanto, a ingestão destas bebidas por indivíduos com predisposição à formação de cálculos renais deve ser comedida.

A presença de água também influencia nas propriedades 
dos sucos de forma que processos de desidratação podem preservar as propriedades nutricionais desses produtos. Assim, além de sucos na forma líquida e de polpa, estes são comercializados também como pó (BIEGAŃSKAMARECIK et al., 2017; KIM, SEONG YEONG, 2017) ou sucos em cápsulas (GORECKI et al., 2018).

Neste sentido, Biegańska-Marecik et al. (2017) desenvolveram um suco de maçã enriquecido com couve congelada e liofilizada e avaliaram o produto em relação à atividade antioxidante pelo ensaio ABTS, o teor de glucosinolato pelo método oficial da UE ISO 9167- 1, conteúdo de ácido ascórbico por HPLC-DAD, compostos fenólicos utilizando detector UV- vis, além das características sensoriais.

Concluíram que a couve liofilizada possui teor de compostos bioativos como glucosinatos (compostos bioativos que desempenham papel importante no metabolismo de células cancerígenas) aumentados em comparação com a couve congelada.

Diversos processos para a produção de sucos em pó têm sido descritos atualmente, tais como o uso de spray-dryer para a produção de suco em pó de beterraba (BAZARIA; KUMAR, 2018), bagaço de frutas vermelhas (REIßNER et al., 2018), pseudo-tronco de banana (SARANYA; SUDHEER, 2017), entre outros. A proporção dos ingredientes dos sucos influencia as características sensoriais e o teor de compostos bioativos.

Para aperfeiçoar uma mistura para suco com propriedades sensoriais e nutricionais, Kim et al. (2017) produziram suco em pó com diferentes razões dos vegetais brócolis, repolho e cenoura em pó, como os principais ingredientes, usando uma proporção fixa de maçã, tomate e rabanete em pó. Foram feitos treze sucos em diferentes proporções. Analisaram o teor de fenólicos total, atividades antioxidantes (ABTS, FRAP) e atributos sensoriais (aceitação geral) como variáveis dependentes. Como consequência, desenvolveram um suco em pó otimizado com alta qualidade sensorial e aumentado nível de compostos fenólicos, o que foi possibilitado pelo aumento da proporção de brócolis e cenoura na mistura.

Alguns sucos apresentaram efeitos sobre a lipoperoxidação e controle de peso. Oliveira et al. (2013) usaram suco verde coado feito de maçã, alface, repolho e pepino e analisaram fenólicos totais, estresse oxidativo (TBARS), superóxido dismutase e catalase no córtex cerebral de ratos. Os ratos foram divididos em três grupos experimentais e submetidos à suplementação por 15 dias, recebendo $5 \mathrm{~mL}$ de suco por quilo de peso. O grupo GJ recebeu suco verde, o grupo OJ, suco de laranja e o grupo controle recebeu água. Os dados mostraram que o suco verde reduziu o ganho de peso e a lipoperoxigenase sugerindo um efeito protetor contra espécies reativas.

Em relação à atividade antioxidante, sucos constituídos por maçã e pepino com adição de especiarias mostraramse efetivos agentes antioxidantes. Babajide et al. (2013) desenvolveram uma bebida funcional com adição de gengibre e analisaram teor de sólidos totais ( $\left.{ }^{\circ} \mathrm{Brix}\right), \mathrm{pH}$ e acidez titulável, gravidade específica, ácido ascórbico (titulação). Analisaram também, por Cromatografia Gasosa acoplada a Espectrometria de Massas (GC-MS), de forma qualitativa, o teor de taninos, flobataninos, saponinas, flavonoides e observaram aumento de fitoquímicos em comparação com a mesma bebida sem adição de gengibre.

\subsubsection{Consumo de bebidas mistas e ação na saúde}

As doenças crônicas não transmissíveis (DCNT) (cardiovasculares, cânceres e diabetes) são responsáveis por cerca de $70 \%$ de todas as mortes no mundo, e a dieta não saudável está entre os principais fatores de risco. Contudo, a alimentação saudável contribui para prevenção dessas doenças (MALTA et al., 2017). No caso destas doenças, o consumo de frutas e vegetais, orientado pelos especialistas em saúde pública, é benéfico para o controle do peso corporal, desde que se mantenha dentro das recomendações diárias, estando diretamente relacionada à prevenção das doenças relacionadas ao excesso de peso (KARIMBEIKI et al., 2018, YU et al., 2018) como hipertensão arterial e hiperlipidemia (ZHENG et al., 2017). Os estudos sobre o efeitos das bebidas na saúde estão no Quadro 3.

Quadro 3 - Estudos sobre a relação entre consumo de bebidas mistas ou seus componentes e a saúde

\begin{tabular}{|c|c|c|}
\hline $\begin{array}{l}\text { Tipo de } \\
\text { Bebida }\end{array}$ & Objetivos & $\begin{array}{l}\text { Autores } \\
\text { (ano) }\end{array}$ \\
\hline Suco líquido & $\begin{array}{l}\text { Verificar se uma dieta com limão } \\
\text { reduziria o peso corporal, a massa } \\
\text { de gordura corporal, a resistência } \\
\text { à insulina e os fatores de risco } \\
\text { para doença cardiovascular. }\end{array}$ & $\begin{array}{c}\text { Kim et al. } \\
\text { (2015) }\end{array}$ \\
\hline $\begin{array}{l}\text { Suco em pó } \\
\text { em cápsula }\end{array}$ & $\begin{array}{l}\text { Verificar a eficácia da } \\
\text { suplementação pré-operatória } \\
\text { com concentrado em pó de suco } \\
\text { de frutas e vegetais encapsulado } \\
\text { para reduzir a morbidade pós- } \\
\text { operatória e melhorar a qualidade } \\
\text { de vida após cirurgia de terceiro } \\
\text { molar inferior. }\end{array}$ & $\begin{array}{l}\text { Gorecki et } \\
\text { al. (2018) }\end{array}$ \\
\hline $\begin{array}{c}\text { Suco líquido } \\
\text { coado }\end{array}$ & $\begin{array}{l}\text { Avaliar os efeitos } \\
\text { antiaterogênicos do consumo de } \\
\text { suco de vegetais no perfil lipídico } \\
\text { e alteração histopatológica em } \\
\text { ratos alimentados com dieta } \\
\text { hiperlipídica. }\end{array}$ & $\begin{array}{c}\text { El- } \\
\text { Shatanovi } \\
\text { et al., } \\
(2012)\end{array}$ \\
\hline $\begin{array}{l}\text { Suco líquido } \\
\text { coado }\end{array}$ & $\begin{array}{l}\text { Verificar o efeito do suco verde } \\
\text { no metabolismo humano por } \\
\text { meio da avaliação de parâmetros } \\
\text { bioquímicos, perfil redox, índice } \\
\text { de massa corporal (IMC) e bem- } \\
\text { estar. }\end{array}$ & $\begin{array}{l}\text { Chiochetta } \\
\text { et al., } \\
\text { (2018) }\end{array}$ \\
\hline
\end{tabular}

Fonte: Dados da pesquisa.

Segundo Chiochetta et al. (2018), a ingestão de suco verde ou com suposta alegação "detox" é popular, mas reitera que não haja um consenso sobre sua composição e efeitos sobre a saúde.

Gorecki et al. (2018) descreveram um suco de hortaliças em pó, veiculado na forma de cápsulas e seu emprego 
em estudo com 183 pacientes em estágio pré-operatório de cirurgia de retirada de terceiro molar. Estes pacientes receberam suplementos deste suco encapsulado ou de placebo por um período relativamente longo (10 semanas no período pré-operatório). Como resultados, os autores relataram que tal suplementação reduziu os efeitos pós-operatórios e a morbidade pós-operatória, contribuindo para melhora na qualidade de vida durante a recuperação após a cirurgia.

O efeito de sucos verde/detox sobre o sobrepeso e obesidade é controverso na literatura. Neste sentido, Kim et al. (2015) avaliaram o efeito detox em mulheres com dieta calórica restritiva. As avaliações de antropometria, sensibilidade à insulina, níveis séricos de adipocinas e marcadores inflamatórios foram realizadas em 84 mulheres coreanas com excesso de peso antes e após o teste de intervenção clínica. Estas foram divididas em três grupos aleatórios: grupo controle sem restrição de dieta, grupo com restrição de dieta e um grupo de dieta detox de limão. O período de intervenção foi de onze dias, dos quais, sete dias ingerindo bebida mista de frutas e vegetais de limão ou suco placebo, seguidos por quatro dias com a transição de alimentos. Foi observada melhora nos níveis de leptina e adiponectina e diminuição no índice de massa corporal e percentual gordura.

Por outro lado, Chiochetta et al. (2018) estudaram o efeito do consumo de suco verde no metabolismo de adulto, divididos em dois grupos (grupo experimental e grupo de controle), com ingestão de $300 \mathrm{~mL}$ de suco durante 60 dias. As avaliações de glicemia, lipídios, funções renais, hepática, enzimas antioxidantes; antropometria, bem-estar e ansiedade foram realizadas e não foram observadas alterações provenientes da dieta preventiva com suco verde.

\section{Conclusão}

As frutas e vegetais, os componentes mais comuns de bebidas mistas são reconhecidos na literatura pela presença de nutrientes e potencial funcional demonstrados em diversos estudos. As bebidas mistas apresentam diferentes formulações e composições nutricionais e podem ter efeitos benéficos sobre a saúde, como foi apontado em estudos in vivo e in vitro. Dentre estes efeitos, destaca-se o potencial antioxidante, que varia de acordo com o tipo e as proporções dos ingredientes que a bebida mista contém, podendo contribuir para elevar o aporte de diferentes micronutrientes. $\mathrm{O}$ tipo de processamento e armazenamento interferem na qualidade sensorial e nutricional do produto. $\mathrm{O}$ consumo de alimentos vegetais $\mathrm{e}$ frutas e, consequentemente, de bebidas preparadas com estes, contribuem para a nutrição e prevenção de doenças sendo um grupo de alimentos importantes na dieta que objetivam uma alimentação saudável. No entanto, diversas propriedades funcionais ainda não são comprovadas, merecendo novos estudos.

\section{Referências}

ALI, B. H et al. Some phytochemical, pharmacological and toxicological properties of ginger (Zingiber officinale Roscoe): A review of recent research. Food Chem. Toxicol., v.46, n.2 p.409420, 2008. doi: 10.1016/j.fct.2007.09.085

ASLANI, N. et al. Effect of garlic and lemon juice mixture on lipid profile and some cardiovascular risk factors in people 30 to 60 years with moderate hyperlipidemia: a randomized controlled trial. Int. J. Prev. Med., v.7, n.1, p.95, 2016. doi: 10.4103/20087802.187248

BABAJIDE, J.M. et al. Physicochemical properties and phytochemical components of spiced cucumber-pineapple fruit drink. Nigerian Food J., v.31, n.1, p.40-52, 2013. doi: 10.1016/ S0189-7241(15)30055-2

BAZARIA, B; KUMAR, P. Optimization of spray drying parameters for beetroot juice powder using response surface methodology (RSM). J. Saudi Soc. Agricul.l Scie., v.17, p.408415, 2018. doi: 10.1016/j.jssas.2016.09.007

BENABDALLAH, A. et al. Total phenolic content and antioxidant activity of six wild Mentha species (Lamiaceae) from northeast of Algeria. Asian Pacific Journal of Tropical Biomedicine, v.6, n.9, p.760-766, 2016. doi: 10.1016/j.apjtb.2016.06.016

BIEGAŃSKA-MARECIK, R.; RADZIEJEWSKA-KUBZDELA, E.; MARECIK, R. Characterization of phenolics, glucosinolates and antioxidant activity of beverages based on apple juice with addition of frozen and freeze-dried curly kale leaves (Brassica oleracea L. var. acephala L.). Food Chem., v.230, p.271-280, 2017. doi: 10.1016/j.foodchem.2017.03.047

BONDONNO, C.P. et al. Flavonoid-rich apples and nitrate-rich spinach augment nitric oxide status and improve endothelial function in healthy men and women: a randomized controlled trial. Free Radical Biology and Medicine, v.52, n.1, p.95-102. 2012. doi: 10.1016/j.freeradbiomed.2011.09.028

BONG, W.C.; VANHANEN, L.P.; SAVAGE, G.P. Addition of calcium compounds to reduce soluble oxalate in a high oxalate food system. Food Chem., v.221, p.54-57, 2017. doi: 10.1016/j. foodchem.2016.10.031

BRASIL. Ministério da Saúde. Secretaria de Atenção à Saúde. Departamento de Atenção Básica. Guia alimentar para a população brasileira. Brasília: MS, 2014.

CAMPÊLO, L. M. L. et al. Antioxidant activity of Citrus limon essential oil in mouse hippocampus. Pharm. Biol., v.49, n.7, p.709-715, 2011. doi: 10.3109/13880209.2010.541924

CANDRAWINATA, V.I. et al. From apple to juice-the fate of polyphenolic compounds. Food Rev. Int., v.29, n.3, p.276-293, 2013. doi: 10.1080/87559129.2013.790049

CARVALHO, A.D.F. et al. A cultura do pepino. Embrapa Hortaliças, p.18, 2013.

CASTILLEJO, N. et al. Microwave heating modelling of a green smoothie: effects on glucoraphanin, sulforaphane and S-methyl cysteine sulfoxide changes during storage. J. Scie. Food Agricul., v.98, n.5, p.1863-1872, 2018. doi: 10.1002/jsfa.8665

CERVO, M.M.C. et al. Effects of canned pineapple consumption on nutritional status, imunomodulation, and physical health of selected school children. J. Nutr. Metabol., v.2014, p.1-9, 2014. doi: 10.1155/2014/861659

CHIOCHETTA, M. et al. Green juice in human metabolism : a randomized trial. J. Am. Coll. Nutr., v.37, n.8, p.670-676, 2018. doi: 10.1080/07315724.2018.1457458

CHUNG, R. W. S. et al. Liberation of lutein from spinach: 
Effects of heating time, microwave- reheating and liquefaction. Food Chem., v.277, p.573-578, 2019. doi: 10.1016/j. foodchem.2018.11.023

DAHMOUNE, F. et al. Valorization of Citrus limon residues for the recovery of antioxidants: Evaluation and optimization of microwave and ultrasound application to solvent extraction. Ind. Crops Prod., v.50, p.77-87, 2013. doi: 10.1016/j. indcrop.2013.07.013

DIFONZO, G. et al. Characterisation and classification of pineapple (Ananas comosus [L.] Merr.) juice from pulp and peel. Food Control, v.96, p.260-270, 2019. doi: 10.1016/j. foodcont.2018.09.015

DIONISIO, A. P. et al. Stability of a functional beverage composed by tropical fruits and yacon (Smallanthus sonchifolius) under refrigerat. Arch.s Latinoam. Nutr., v.66, n.2, p.148-155, 2016.

DUZZIONI, A. G. et al. Determinação da atividade antioxidantes e de constituintes bioativos em frutas cítricas. Aliment. Nutr. Araraquara, v.21, n.4, p.643-649, 2010.

EBRAHIMZADEH ATTARI, V. et al. Changes of serum adipocytokines and body weight following Zingiber officinale supplementation in obese women: a RCT. Euro. J. Nutr., v.55, n.6, p.2129-2136, 2016. doi: 10.1007/s00394-015-1027-6

EL-SHATANOVI, G.A.T.A. et al. Antiatherogenic properties of vegetable juice rich in antioxidants in cholesterol-fed rats. Ann. Agricul. Scie., v.57, n.2, p.167-173, 2012. doi: 10.1016/j. aoas.2012.08.011

ERCOLI, L. et al. Evaluation of chemical characteristics and correlation analysis with pulp browning of advanced selections of apples grown in Brazil. Acta Scie. Technol., v.39, n.1, p.103, 2017. doi: 10.4025/actascitechnol.v39i1.27913

FERRENTINO, G. et al. Biorecovery of antioxidants from apple pomace by supercritical fluid extraction. J. Cleaner Prod., v.186, p.253-261, 2018. doi: 10.1016/j.jclepro.2018.03.165

FIORITO, S. et al. Novel biologically active principles from spinach, goji and quinoa. Food Chemistry, v.276, p.262-265, 2019. doi: 10.1016/j.foodchem.2018.10.018

GONZÁLEZ-MOLINA, E. et al. Natural bioactive compounds of Citrus limon for food and health. J. Pharm. Biom. Anal., v.51, n.2, p.327-345, 2010. doi: 10.1016/j.jpba.2009.07.027

GORECKI, P. et al. Perioperative supplementation with a fruit and vegetable juice powder concentrate and postsurgical morbidity: a double-blind, randomised, placebo-controlled clinical trial. Clin. Nutr., v.37, n.5, p.1448-1455, 2018.

HAJAR, N. et al. Physicochemical properties analysis of three indexes pineapple (ananas comosus) peel extract variety n36. APCBEE Proc., v.4, p.115-121, 2012.

HAN, J. H. et al. The effect of glutathione S-transferase M1 and T1 polymorphisms on blood pressure, blood glucose, and lipid profiles following the supplementation of kale (Brassica oleracea acephala) juice in South Korean subclinical hypertensive patients. Nutr. Res. Pract., v.9, n.1, p.49-56, 2015. doi: 10.4162 / nrp.2015.9.1.49.

HODGES, R.E.; MINICH, D.M. Modulation of metabolic detoxification pathways using foods and food-derived components: a scientific review with clinical application. J. Nutr. Metabol., v.2015, p.1-23, 2015. doi: 10.1155/2015/760689

HORNEDO-ORTEGA, R. et al. Influence of fermentation process on the anthocyanin composition of wine and vinegar elaborated from strawberry. J. Food Scie., v.82, n.2, p. 364-372, 2017. doi: $10.1111 / 1750-3841.13624$
HOSSAIN, M. A.; RAHMAN, S. M. M. Total phenolics, flavonoids and antioxidant activity of tropical fruit pineapple. Food Res. Int., v.44, n.3, p.672-676, 2011. doi: 10.1016/j. foodres.2010.11.036

JAISWAL, S.K. et al. Hepatoprotective Effect of Citrus limon Fruit Extract against Carbofuran Induced Toxicity in Wistar Rats. Chin. J. Biol., v.2015, p.1-10, 2015. doi: 10.1155/2015/686071

JAYAPRAKASHA, G. K. et al. Chemical composition of volatile oil from Citrus limettioides and their inhibition of colon cancer cell proliferation. Ind. Crops Prod., v.45, p.200-207, 2013. doi: 10.1016/j.indcrop.2012.12.020

JEON, J. et al. Transcriptome analysis and metabolic profiling of green and red kale (Brassica oleracea var. acephala) seedlings. Food Chem., v.241, p.7-13, 2018. doi: 10.1016/j. foodchem.2017.08.067

KALINOWSKA, M. et al. Apples: Content of phenolic compounds vs. variety, part of apple and cultivation model, extraction of phenolic compounds, biological properties. Plant Physiol. Biochem., v.84, p.169-188, 2014. doi: 10.1016/j. plaphy.2014.09.006

KARGUTKAR, S.; BRIJESH, S. Anti-inflammatory evaluation and characterization of leaf extract of Ananas comosus. Inflammopharmacol., v.26, n.2, p.469-477, 2018. doi: 10.1007/ s10787-017-0379-3

KARIMBEIKI, R. et al. Higher dietary diversity score is associated with obesity: a case-control study. Public Health, v.157, p.127-134, 2018. doi: 10.1016/j.puhe.2018.01.028

KHAN, R. A.; RIAZ, A. Behavioral effects of citrus limon in rats. Metabol. Brain Dis., v.30, n.2, p.589-596, 2015. doi: 10.1007/ s11011-014-9616-2

KHONCHE, A. et al. Efficacy of Mentha pulegium extract in the treatment of functional dyspepsia: A randomized double-blind placebo-controlled clinical trial. Journal of Ethnopharmacology, v.206, p.267-273, 2017. doi: 10.1016/j.jep.2017.05.026

KIM, M-B; KO, J-Y; LIM, S-B. Formulation optimization of antioxidant-rich juice powders based on experimental mixture design. J. Food Proc. Preserv., v.41, n.3, p.1-10, 2016. doi: 10.1111/jfpp.12897

KIM, M. J. et al. Lemon detox diet reduced body fat, insulin resistance, and serum hs-CRP level without hematological changes in overweight Korean women. Nutr. Res., v.35, n.5, p.409-420, 2015. doi: 10.1016/j.nutres.2015.04.001

KIM, S.Y. Production of fermented kale juices with lactobacillus strains and nutritional composition. Prev. Nutr. Food Scie., v.22, n.3, p.231-236, 2017. doi: 10.3746/pnf.2017.22.3.231

KLEIN, A. V.; KIAT, H. Detox diets for toxin elimination and weight management: a critical review of the evidence. J. Hum. Nutr. Diet., v.28, n.6, p.675-686, 2015. doi: 10.1111/jhn.12286

KUSHIMOTO, S. et al. Kale supplementation up-regulates HSP70 and suppresses cognitive decline in a mouse model of accelerated senescence. J. Funct. Food, v.44, p.292-298, 2018. doi: 10.1016/j.jff.2018.03.011

LIMA, A. et al. Ginger (Zingiber Officinale Roscoe), Bioactive properties and its possible effect on type 2 Diabetes: a review study. Rev. Saúde Foco, v.1, n.2, p.15-25, 2014.

LIN, L. Z.; HARNLY, J. M. Identification of the phenolic components of collard greens, kale, and chinese Broccoli. $J$. Agric. Food Chem., v.57, n.16, p.7401-7408, 2009. doi: 10.1021/ jf901121v

MALTA, D. C. et al. Noncommunicable diseases and the use of health services: Analysis of the National Health Survey in Brazil. 
Rev. Saude Publica, v.51, p.1S-10S, 2017. doi: 10.1590/s15188787.2017051000090

MARTÍN, I. S. M. et al. Are detox diets an effective strategy for obesity and oxidation management in the short term? J. Negative Positive Results, v.2, n.9, p.399-409, 2017. doi: 10.19230/ jonnpr. 1585

MOURA, S.C.S.R. et al. Characterization and evaluation of stability of bioactive compounds in fruit smoothies. Food Scie. Technol., v.37, n.2, p.216-223, 2017. doi:10.1590/1678$457 x .16616$

MUKHERJEE, P. K. et al. Phytochemical and therapeutic potential of cucumber. Fitoterapia, v.84, n.1, p.227-236, 2013. doi: 10.1016/j.fitote.2012.10.003

NASH, R. J. et al. Effectiveness of Cucumis sativus extract versus glucosamine-chondroitin in the management of moderate osteoarthritis: a randomized controlled Trial. Clin. Interv. Aging, v.13, p.2119-2126, 2018. doi: 10.2147/CIA.S173227

NAYEBIFAR, S et al. The effect of a 10-week high-intensity interval training and ginger consumption on inflammatory indices contributing to atherosclerosis in overweight women. J. Res. Med. Scie., v.21, n.8, p.1-7, 2016. doi: 10.4103/1735-1995.193507

NRIPENDRA, N.B.; SAHA, S.; ALI, M.K. Comparative studies on the in vitro antioxidant properties of methanolic leafy extracts from six edible leafy vegetables of India. Asian J. Pharm. Clin. Res., v.6, n.3, p.96-99, 2013. doi: 10.12980/APJTB.4.2014C1298

OBERT, J. et al. Popular weight loss strategies: a review of four weight loss techniques. Current Gastroenterol. Reports, v.19, n.12, p.17-20, 2017. doi: 10.1007/s11894-017-0603-8

OLIVEIRA, P.S. et al. Green juice a protector against reactive species in rats. Nutr. Hosp., v.28, n.5, p.1407-1412, 2013. doi: 10.3305/nh.2013.28.5.6505

REIßNER, A. et al. Composition and physicochemical properties of dried berrypomace. J. Scie. Food Agricul., v.99, p.1284-1293, 2018. doi: $10.1002 /$ jsfa.9302

REIS, N. S. et al. Uma abordagem sobre o potencial funcional das diferentes matrizes vegetais; alho, menta e gengibre. Rev. Bras. Ciênc. Saúde, v.1, p.82-91, 2017.

ROONEY, C. et al. How much is '5-a-day'? A qualitative investigation into consumer understanding of fruit and vegetable intake guidelines. J. Hum. Nutr. Dietetics, v.30, n.1, p.105-113, 2017. doi: $10.1111 / \mathrm{jhn} .12393$

SAMPATH, C. et al. Specific bioactive compounds in ginger and apple alleviate hyperglycemia in mice with high fat diet-induced obesity via Nrf2 mediated pathway. Food Chem., v.226, p.79-88, 2017. doi: 10.1016/j.foodchem.2017.01.056
SARANYA, S; SUDHEER, K.P. Development of fortified banana pseudostem juice powder utilizing spray drying technology. $J$. Trop. Agric., v.55, n.2, p.145-151, 2017. doi: 10.1157/13098492

SNYDER, A.B.; WOROBO, R.W. The incidence and impact of microbial spoilage in the production of fruit and vegetable juices as reported by juice manufacturers. Food Control, v.85, p.144150, 2018. doi: 10.1016/j.foodcont.2017.09.025

SPÍNOLA, V.; PINTO, J.; CASTILHO, P.C. In vitro studies on the effect of watercress juice on digestive enzymes relevant to type 2 diabetes and obesity and antioxidant activity. J. Food Biochem., v.41, n.1, p.1-8, 2017. doi: 10.1111/jfbc.12335

SRIDHARAN, B. et al. Beneficial effect of Citrus limon peel aqueous methanol extract on experimentally induced urolithic rats. Pharm. Biol., v.54, n.5, p.759-769, 2016. doi: 10.3109/13880209.2015.1079724

SYAMILA, M. et al. Effect of temperature, oxygen and light on the degradation of $\beta$-carotene, lutein and $\alpha$-tocopherol in spraydried spinach juice powder during storage. Food Chem., v.284, p.188-197, 2019. doi: 10.3109/13880209.2015.1079724

THAVARAJAH, D. et al. Mineral micronutrient and prebiotic carbohydrate profiles of USA- grown kale (Brassica oleracea L. var. acephala). J. Food Comp. Anal., v.52, p.9-15, 2016. doi: 10.1016/j.jfca.2016.07.003

VANHANEN, L.; SAVAGE, G. Comparison of oxalate contents and recovery from two green juices prepared using a masticating juicer or a high-speed blender. NFS J., v.1, p.20-23, 2015. doi:10.1016/j.nfs.2015.07.002

VIDAL, N. P. et al. The use of natural media amendments to produce kale enhanced with functional lipids in controlled environment production system. Scie. Reports, v.8, n.1, p.1-14, 2018. doi: 10.1038/s41598-018-32866-5

WRUSS, J. et al. Differences in pharmacokinetics of apple polyphenols after standardized oral consumption of unprocessed apple juice. Nutr. J., v.14, n.1, p.32, 2015. doi:10.1038/s41598018-32866-5

YU, M. et al. Rapid analysis of benzoic acid and vitamin $\mathrm{C}$ in beverages by paper spray mass spectrometry. Food Chem., v.268, p.411-415, 2018. doi: 10.1016/j.ijms.2018.10.003

$\mathrm{ZHAO}$, L. et al. $\mathrm{CeO} 2$ and $\mathrm{ZnO}$ nanoparticles change the nutritional qualities of cucumber (Cucumis sativus). J. Agricul. Food Chem., v.62, n.13, p.2752-2759, 2014. doi: 10.1016/j. foodchem.2018.11.114

ZHENG, J. et al. Effects and Mechanisms of Fruit and Vegetable Juices on Cardiovascular Diseases. Int. J. Mol. Scie., v.18, n.3, p.555, 2017. doi: 10.3390/ijms 18030555 